\title{
Shortwave infrared detection of medical radioisotope Cerenkov luminescence
}

\author{
Jan Grimm ( $\nabla$ grimmj@mskcc.org ) \\ Benedict Mc Larney \\ Memorial Sloan Kettering Cancer Center \\ Qize Zhang \\ Memorial Sloan Kettering Cancer Center

\section{Edwin Pratt} \\ Memorial Sloan Kettering Cancer Center \\ Magdalena Skubal \\ Memorial Sloan Kettering Cancer Center \\ Elizabeth Isaac \\ Memorial Sloan Kettering Cancer Center \\ Hsiao-Ting Hsu \\ Memorial Sloan Kettering Cancer Center \\ Anuja Ogirala \\ Memorial Sloan Kettering Cancer Center
}

Memorial Sloan Kettering Cancer Center https://orcid.org/0000-0002-5282-9385

\section{Article}

Keywords:

Posted Date: February 2nd, 2022

DOI: https://doi.org/10.21203/rs.3.rs-1321954/v1

License: (c) (i) This work is licensed under a Creative Commons Attribution 4.0 International License.

Read Full License 

Benedict E. Mc Larney ${ }^{1,2}$, Qize Zhang ${ }^{1,2}$, Edwin C. Pratt ${ }^{1,2}$, Magdalena Skubal ${ }^{1,2}$, Elizabeth Isaac $^{1,2}$, Hsiao-Ting Hsu ${ }^{1,2}$, Anuja Ogirala ${ }^{1,2}$, Jan Grimm ${ }^{1,2,3,4,5,{ }^{*}}$

1. Molecular Pharmacology Program, Memorial Sloan Kettering Cancer Center, New York, NY, USA

2. Molecular Imaging Therapy Service, Memorial Sloan Kettering Cancer Center, New York, NY, USA

3. Pharmacology Program, Weill Cornell Medical College, New York, NY USA

4. Department of Radiology, Memorial Sloan Kettering Cancer Center, New York, NY, USA

5. Department of Radiology, Weill, Cornell Medical Center, New York, NY, USA

* . Corresponding author: Jan Grimm - grimmi@mskcc.org

\begin{abstract}
Cerenkov luminescence $(C L)$ is produced by medical radioisotopes when charged (commonly beta $\left(\beta^{+-}\right)$) particles travel faster than light in a dielectric medium (tissue). This blue-weighted luminescence is both continuous and proportional to the reciprocal wavelength. CL imaging (CLI) promises an economical alternative to PET but is limited by the optical properties of tissue and special setup requirements. CL has been detected in the shortwave infrared (SWIR) spectrum $(900-1700 \mathrm{~nm})$ from linear accelerators operating in the MeV range but so far not from medical radioisotopes. This work is the first to show that the order of magnitude weaker SWIR CL from medical radioisotopes predicted by the Frank-Tamm equation can also be detected, using commercially available SWIR components. SWIR CL was detected from five clincial radioisotopes: ${ }^{90} \mathrm{Y},{ }^{68} \mathrm{Ga},{ }^{18} \mathrm{~F},{ }^{89} \mathrm{Zr},{ }^{131} \mathrm{I}$ and from ${ }^{32} \mathrm{P}$, used in biomedical research. The advantage of radioisotope SWIR CLI over conventional CLI is shown in terms of significantly increased light penetration and reduced scattering at tissue depth, in line with the known advantages of SWIR imaging. We report the radioisotope SWIR spectrum, the current detection sensitivity limit $(0.23$ $\mu \mathrm{Ci} / \mu \mathrm{l}$ of ${ }^{68} \mathrm{Ga}$ ) and determine the feasibility of SWIR CLI with ex vivo and in vivo preclinical examples. Further improvements in SWIR optics and technology are required to enable widespread adoption.
\end{abstract}

\title{
1. Introduction
}

$\mathrm{CL}$ is generated by subatomic suprarelativistic particles (i.e., traveling faster than the speed of light) in a dielectric medium such as water or tissue.[1] As the particle travels it polarizes the surrounding molecules, which generate light (luminescence) upon relaxation [2]. CL is UV weighted, appearing blue, with an exponential decrease in photon production as the wavelength increases $\left(1 / \lambda^{2}\right)$ throughout the visible and into the infrared spectrum.[3] The detected CL intensity is directly correlated to the energy of the emitted particle upon decay of the isotope and has found a variety of uses in astrophysics, nuclear physics, and more recently in biomedic imaging. $[4,5]$

Cerenkov luminescence imaging (CLI) has been established as a cost- and time-effective alternative to positron emission tomography (PET), especially for surface-weighted imaging.[2, 6] Biomedical CLI has focused on two different entitites: the detection of CL from radioisotopes decaying with a beta $\left(\beta^{+/}\right)$particle emission used in nucelar medicine; and from a linear accelerator (LINAC) in the pre- and clinical domain.[7, 8] The advantages of using inexpensive (in comparison to PET) optical based systems to detect signals from specific radiotracers has aided in the discovery and development of both novel targeted radiotracers and radiotherapy- 
based treatments.[9] The success of preclinical CLI has led to the implementation of clinical CLI. To date, clinical CLI has focused on image guided surgery for margin detection, determining the clinical uptake of radiotracers and real time dosimetry readings.[10-13] Numerous clinically approved and commonplace radioisotopes have been shown to produce detectable $\mathrm{CL}$ with standard administered doses.[4]

The rapid adoption of devices capable of detecting single photons of light by the life science community has resulted in a wide selection of affordable and low dark and read noise optical devices. Fortunately these cameras, based on EMCCD and scientific CMOS technology, are highly sensitive to the blue weighted CL spectrum.[2] Whilst visible wavelengths of light are often desirable in both fluorescence and microscopy-based imaging, these wavelengths suffer significant drawbacks in both the pre- and clinical settings. The abundance of endogenous chromophores in biological tissue limits the optical detection of $C L$ in the visible parts of the spectrum to penetration depths of a few millimeters.[14] Furthermore, light only remains within its ballistic regime for a few hundred microns in tissue before entering the scattering or random walk regime.[15] This greatly reduces the resolution, contrast and ultimate sensitivity of visible light imaging at increased depth. Accordingly, CLI is limited by the same optical properties of tissue.

As the limitations of visible light (VIS)-based imaging have been defined, there has more recently been a noted shift to longer wavelength-based imaging where the absorption and scattering of light is reduced.[16] These methods have focused on imaging in the near infrared region (NIR) above $650 \mathrm{~nm}$ where tissue absorption is reduced by almost two orders of magnitude compared to visible wavelengths $(400-650 \mathrm{~nm})$. [14, 17] Accordingly, numerous studies have focused on the red shifting conversion of $C L$ via dyes and nanoparticles.[18-20] Of note is the more recent emergence of short-wave infrared (SWIR; $900-1700 \mathrm{~nm}$ ) based imaging where tissue absorption, scattering and autofluorescence are of negligible levels.[21] The advantages of SWIR imaging have been outlined in many cases including the imaging of the clinically approved dye ICG with increased resolution and contrast over VIS and NIR wavelengths.[22] Recent examples have shown the detection of SWIR photons from nanoprobes excited by X-ray beams and fluorescent emission of quantum dots excited by CL from an external LINAC beam in a FRET manner.[18, 23-25] Furthermore, pure CLI without the need for secondary emitters has been detected from LINAC sources where significant improvements were shown by SWIR CL versus VIS-NIR CL.[26] In comparison to LINAC based CLI, imaging of medical radioisotopes CL is more demanding. On average, radioisotopes produce an order of magnitude less energy than LINAC particles achieve (6 to $24 \mathrm{MeV}$ from LINACs versus $0.836 \mathrm{MeV}$ for e.g. ${ }^{68} \mathrm{Ga}$, one of the brightest $\mathrm{CL}$ medical radioisotopes).[27-30] The low $\mathrm{CL}$ production from radioisotopes requires complete darkness during imaging in combination with low noise and highly efficient optical imaging systems. The low luminescence production of radioisotopes in comparison to LINACs additionally increases the difficulty for radioisotope SWIR CL detection.[3] Furthermore, the acquisition of CL generated by a LINAC can be synchronized with the radiation pulses for better sensitivity, something not possible for radioisotope decay.[28, 31] Despite these challenges, we show here for the first time that SWIR CLI can be performed with medical radioisotopes with significant advatanges over VIS CLI.

Currently, commercial SWIR technology is far less advanced than its visible light EMCCD counterparts in terms of both sensor read and dark charge noise. Thermoelectric cooled (TEC) EMCCDs can readily achieve dark noise levels of $0.001 \mathrm{e} / \mathrm{p} / \mathrm{s}$ in comparison to values ranging from 40 to $300 \mathrm{e} / \mathrm{p} / \mathrm{s}$ for TEC cooled SWIR sensors. Additionally, high efficiency visible light collecting optics are commercially available with an $f$ of 0.95 . This is in stark comparison to commercially available SWIR lenses which currently achieve an $f$ of only 1.4 , collecting less than half the light of 0.95 lenses. The low light output of radioisotope $C L$, orders of magnitude increased 
SWIR dark and read sensor noise in combination with less sensitive lenses further exacerbates the challenge to detect radioisotope SWIR CL. In this work, radioisotope SWIR CLI was achieved via specialized TEC SWIR cameras, appropriate acquisition settings and post acquisition processing. Herein we characterize the performance of SWIR CL with radioisotopes, detect SWIR $\mathrm{CL}$ emission from six radioisotopes, define the SWIR CL radioisotope emission spectrum, show the advantage of SWIR CLI over VIS CLI, define the current limit of detection in terms of sensitivity and assess the feasibility of preclinical radioisotope SWIR CL in both ex vivo and in vivo settings. This work establishes a proof of principle for radioisotope SWIR CLI. We also highlight the need for improved SWIR sensors and optics to mature SWIR CLI technology to a level seen with VIS CLI.

\section{Results}

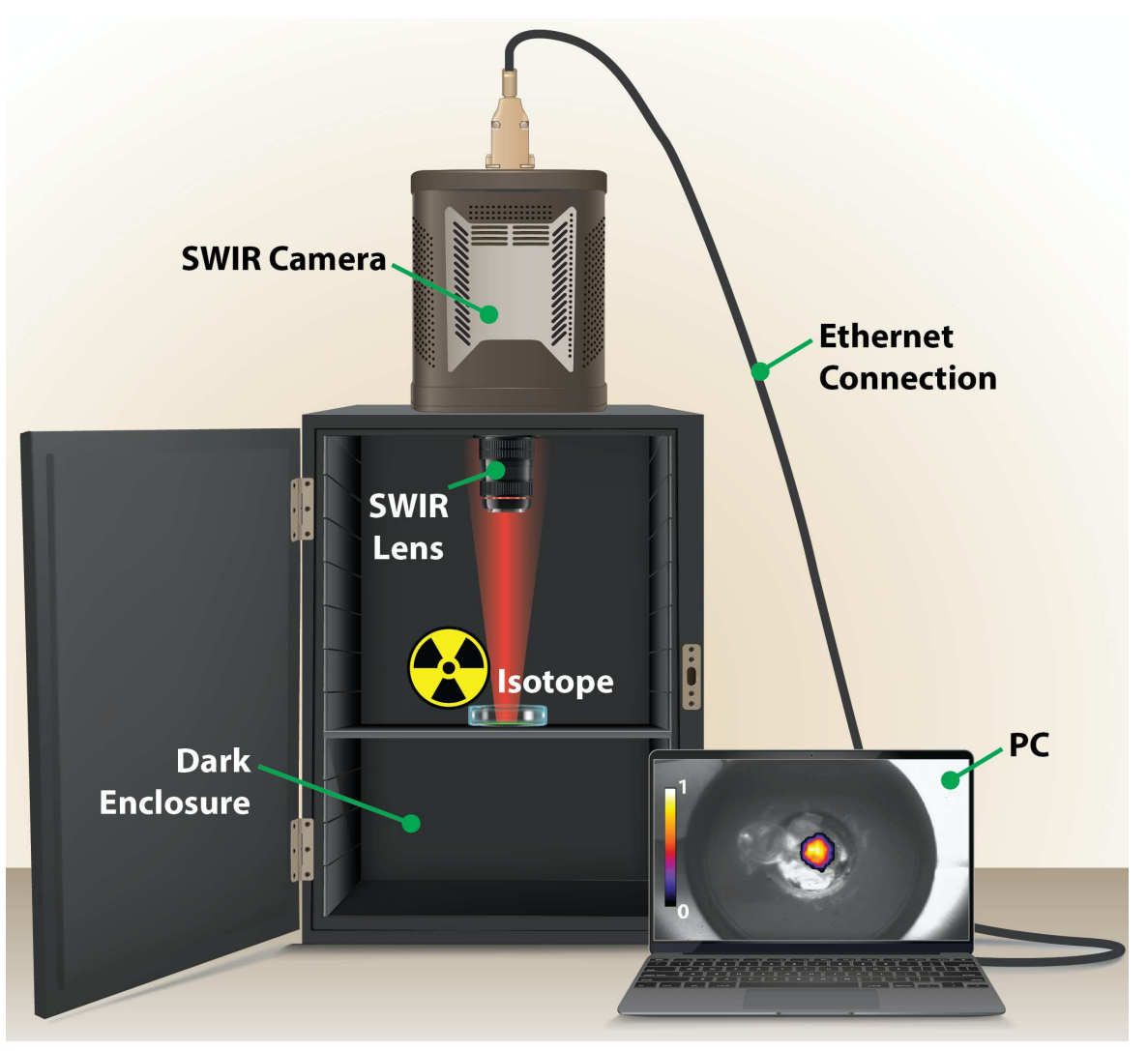

Figure 1. Schematic outlining the SWIR CL radioisotope imaging setup. Imaging was carried out in a custom dark enclosure with the radioisotope source located within a lead ingot (pig) for shielding. The camera was fitted with either a $16 \mathrm{~mm}$ or $8 \mathrm{~mm}$ f/1.4 SWIR lens and located $20 \mathrm{~cm}$ from the source with a field of view of $15 \times 15 \mathrm{~cm}$. Camera control and recordings were managed from the respective camera software on a PC.

\subsection{Confirmation of SWIR CL detection from radioisotopes}

${ }^{68} \mathrm{Ga}$ is a commonly administered medical radioisotope for PET imaging.[32, 33] During its decay, ${ }^{68} \mathrm{Ga}$ produces $\beta^{+}$particles $(87.7 \%)$ along with $y$ photons. As shown in Figure $2 \mathrm{~A}$, the SWIR CLI setup (see Figure $1 \mathrm{~A}$ ) could readily detect the SWIR radioisotope $\mathrm{CL}$ emission. A sample of ${ }^{68} \mathrm{Ga}$ $(2.95 \mathrm{mCi}$ ) was eluted into a $5 \mathrm{ml}$ Eppendorf and placed in a lead pig within the field of view (FOV). 
Shown in Figure 2A top right is the processed SWIR CL image overlaid on the white light image demonstrating that the source of light is coming from the Eppendorf and that the processing steps sufficiently removed $y$ strikes on the camera chip whilst retaining the intensity of the light source. To ensure that the image processing did not accumulate $y$ strikes to resemble the light source, a piece of black cardboard was placed over the source. As shown in Figure $2 \mathrm{~A}$, the cardboard image resulted in a blank image confirming that the images are based on the the detection of SWIR CL photons and not y strike accumulation. The ${ }^{68} \mathrm{Ga}$ source was then moved around the FOV decaying in the process (five positions in total including a repeat of $\mathrm{P} 1$ ). The sensor could readily detect $\mathrm{CL}$ from ${ }^{68} \mathrm{Ga}$ across the entire FOV. Manually drawn regions of interest (ROI) were 
intervals. The Pearson correlation value is displayed $\left(R^{2}=0.9839\right)$, two-tailed $p$ value $<0.0001$ highlighting the conserved quantitative and linear respone capabilities of SWIR CLI, in line with VIS CLI. Each image and data point are a summation of $n=90$ technical replicates for each position. Presented images are thresholded at the same levels to show lowest light levels, allowing noise and sensor artifacts to show at 1.32 and $1.09 \mathrm{mCi}$.

As can be seen in Figure 2B the recorded gray levels increase in a manner that is linearly correlated with the ${ }^{68} \mathrm{Ga} \mathrm{mCi} \mathrm{levels} \mathrm{highlighting} \mathrm{the} \mathrm{accuracy} \mathrm{and} \mathrm{quantitative} \mathrm{capabilties} \mathrm{of} \mathrm{SWIR}$ CLI setup at high radioisotope levels. This linearity is in line with VIS CLI.[4] As is common with optical imaging devices, linearity and sensitivity are reduced along imaging borders as shown by the artefacts at P4 and P5 in Figure 2.

As SWIR CLI could be performed with ${ }^{68} \mathrm{Ga}(0.84 \mu \mathrm{Ci} / \mu \mathrm{l})$, we set out to investigate other radioisotopes which may produce enough SWIR CL to be detected using our setup. As shown in Figure $3 \mathrm{~A}$, four additional radioisotopes were detected: ${ }^{32} \mathrm{P}(8.65 \mu \mathrm{Ci} / \mu \mathrm{l}),{ }^{18} \mathrm{~F}(272.25 \mu \mathrm{Ci} / \mu \mathrm{l}),{ }^{89} \mathrm{Zr}$ $(160 \mu \mathrm{Ci} / \mu \mathrm{l})$ and ${ }^{131} \mathrm{I}(1112 \mu \mathrm{Ci} / \mu \mathrm{l})$. The same processing steps as used for the ${ }^{68} \mathrm{Ga}$ imaging were employed to ensure that the detected signals were not as a result of $\gamma$ strikes and correctly overlaid with the white light image of the source location. In all cases the CL was correctly overlaid with the position of the source, see Figure 3A. The SWIR radiance of each was then calculated as shown in Figure 3B, values have been corrected for both concentration $(\mu \mathrm{Ci} / \mu \mathrm{l})$ and spatial FOV $\left(\mathrm{cm}^{2}\right)$. SWIR CLI could readily differentiate between each of the tested radioisotopes, as shown by the $p$ values in Figure 3B. The reported SWIR CL levels are in line with previously reported VIS CLI values highlighting the continuity of the technique.[34]
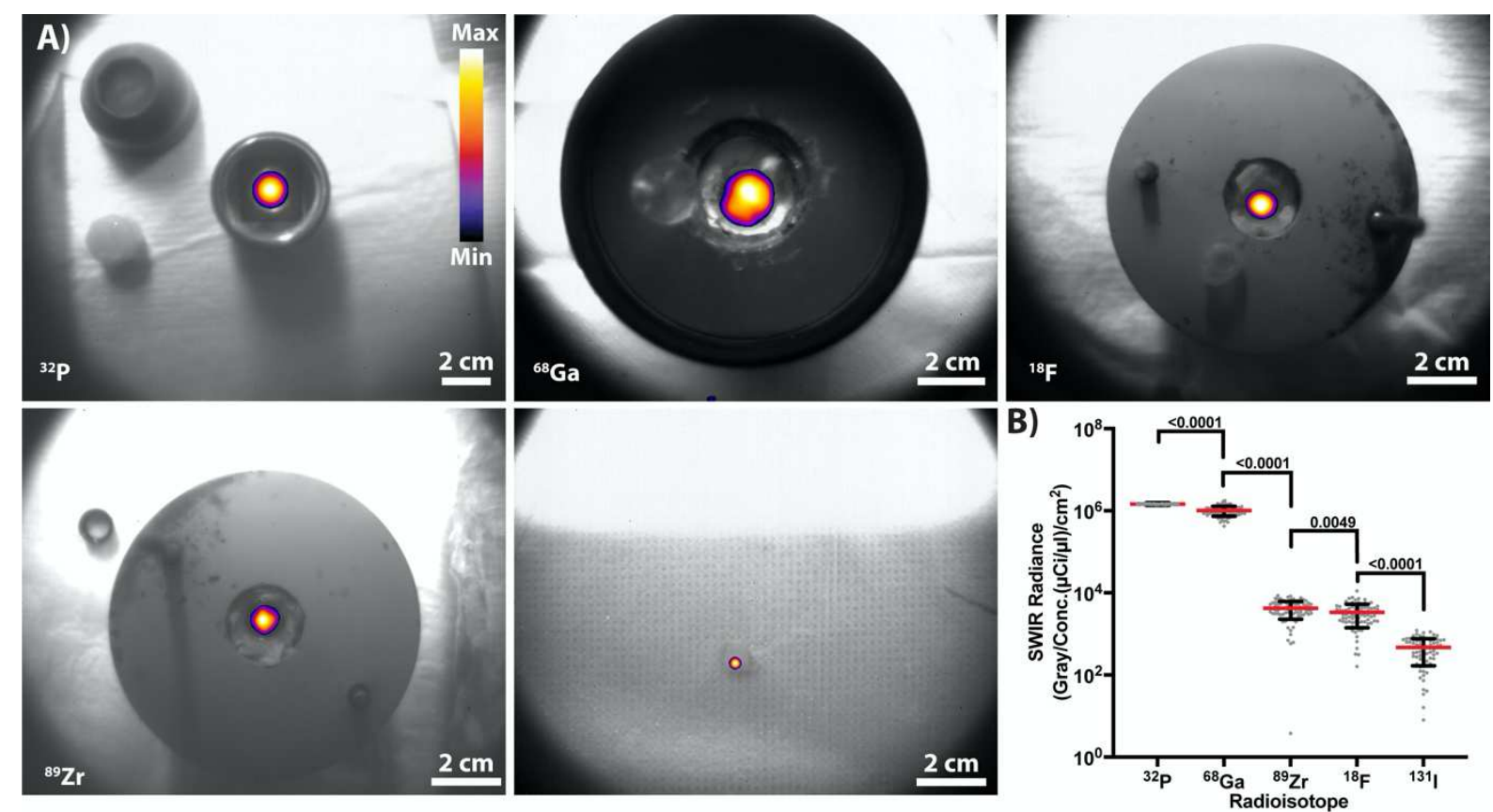

Figure 3. SWIR CLI of a variety of medical radioisotopes. A) Top and bottom, representative images are shown for SWIR CLI detection of ${ }^{32} \mathrm{P},{ }^{68} \mathrm{Ga},{ }^{18} \mathrm{~F},{ }^{89} \mathrm{Zr}$ and ${ }^{131} \mathrm{l}$. Each image is thresholded respectively and represents the summation of $n=90$ technical replicates. B) Comparative descending radiance of the isotopes which have been corrected for concentration $(\mu \mathrm{Ci} / \mu \mathrm{l})$ and spatial FOV. Students t-test (upaired, two-sided) $p$ values are shown highlighting the systems capability to distinguish between radioisotopes. The mean (red line) and standard deviation are shown. Individual measurements ( $n=90$ technical replicates) are displayed with gray dots for each sample, excluding negative values. 
182

183

184

185

186

187

188

189

190

191

192

193

194

195

196

197

198

199

200

201

202

203

204

205

206

207

208

\subsection{Determining the SWIR CLI radioisotope temporal detection limit and emission spectrum}

${ }^{32} \mathrm{P}$ is a pure $\beta^{-}$particle emitter with a long half-life of 14.3 days and provides a stable source for setup characterization. The ${ }^{32} \mathrm{P}$ concentrations used here ensured SWIR CLI detection with signal being distinguished from noise at acquisitions speeds as low as $0.25 \mathrm{~s}$, see Figure 4A. Each image panel of Figure 4A represents the summation of 90 frames at each respective exposure time. Figure 4B shows the mean gray level along with each of the 90 repetitions at the respective acquisition length.

The high SWIR CL radiance of ${ }^{32} \mathrm{P}$ was then used to characterize the radioisotope SWIR CL emission spectrum. No filter (wavelengths of $920 \mathrm{~nm}$ and above due to the spectral response of the sensor) and long pass filtered acquisitions ranging from $1000 \mathrm{~nm}$ to $1500 \mathrm{~nm}$ in $100 \mathrm{~nm}$ steps were carried out. The sensor used in these experiments employs a indium phosphide ( $\operatorname{lnP})$ substrate band gap at $1.35 \mathrm{eV}$. Whilst InGaAs itself can detect VIS light, the InP band gap is not thinned on this device and thus prevents light below $920 \mathrm{~nm}$ from reaching the sensor. Additionally, InGaAs focal plane arrays have an inherent bandgap of $0.75 \mathrm{eV}$ resulting in a shortpass cutoff at 1700nm.[35-37] The images of the SWIR CL photon production are shown in Figure $4 \mathrm{C}$ with the intensity exponentially decreasing as the wavelength increased, as expected and previously reported for VIS CLI and LINAC based SWIR CLI.[2, 26] The radioisotope SWIR $C L$ spectrum was then quantified as shown in Figure 4D showing a fitted one phase exponential decay with an $R^{2}$ of 0.9812 . As can be seen in Figure $4 C$ and $D$ detection of photons above $1400 \mathrm{~nm}$ is challenging with the current setup which can likely be attributed to the noise within the system as opposed to purely low lumiescence production. The noise is highlighted by the noise floor level shown in Figure 4D.
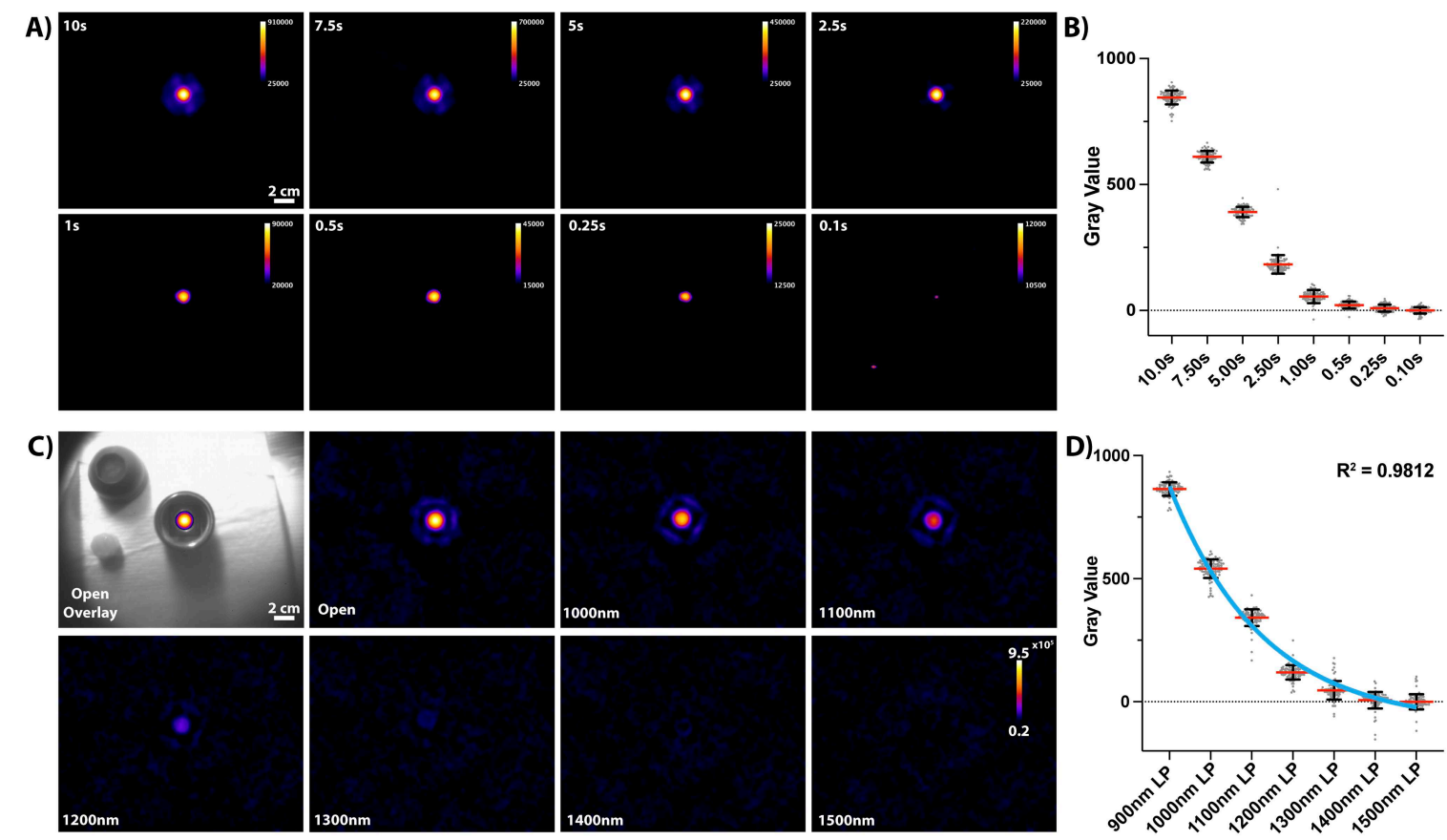

Figure 4. Determining the radioisotope SWIR CLI temporal acquisition limit and spectrum A) Representative images of the effect of exposure time changes on image quality for each exposure length. SWIR CL could be separated from the noise at acquisition speeds of up to $0.25 \mathrm{~s}$. B) The gray 
value intensity as exposure time changes. For A) each image is a summation of $n=90$ technical replicates and $B$ ) is representative of each replicate where the mean and standard deviation are displayed. C) The SWIR CL emission spectrum of ${ }^{32} P$ is shown. Firstly, overlaid and then by images for each filter set. The output decreases as the wavelength increases. Each image is a summation of $n=90$ technical replicates. D) Graphical representation of the radioisotope SWIR CL emission spectrum from 920 to $1500 \mathrm{~nm}$, mean and standard deviation are displayed from $n=90$ technical replicates. A one phase exponential decay function has been fitted $\left(R^{2}=0.9812\right)$. As shown in $\left.C\right)$ the inherent noise in the system and low photon production prevents detection of photons above 1400 $n m$.

\subsection{Reduced scattering via SWIR CLI over VIS CLI}

Having established suitable acquisition and post processing steps, we then investigated the advantage of SWIR over VIS CLI. To achieve this an eppedorf containing $1.5 \mathrm{mCi}$ of ${ }^{90} \mathrm{Y}$ suspended in $200 \mu \mathrm{l}$ of saline solution was imaged on the SWIR setup and immediately after on a current state of the art IVIS ${ }^{\circledR}$ (VIS CLI) imaging system. ${ }^{90} \mathrm{Y}$ is a pure $\beta^{-}$emitter (only CL) with a $64 \mathrm{~h}$ half life that is commonly administered clinically to treat primary and metastatic liver cancer at activities ranging from 13.5 to $143 \mathrm{mCi} .[38,39]$ Representative images of the source with and without overlying scattering medium (chicken breast) were acquired on the SWIR and IVIS systems at increasing tissue depths of 0,10 and $15 \mathrm{~mm}$. To enable a fair comparison between both modalities the imaging time was defined as the minimum time needed to detect signal from the source without any scattering medium, $10 \mathrm{~s}$ for IVIS imaging and 15 mins for SWIR CLI. These exposure times were consistent throughout the increase in tissue depth. As can be seen in Figure 5 , SWIR CLI shows an improvement in resolution when imaging through scattering tissue. Observationally, for SWIR CLI the shape of the Eppendorf containing ${ }^{90} \mathrm{Y}$ remains consistent up to $15 \mathrm{~mm}$ of tissue whilst its appearance is enlargened over five times in VIS CLI. This highlights the advantage of SWIR CLI over VIS CLI to accurately resolve radioisotope's location at depth in scattering media like tisse. This accuracy is further validated by the full width half maximum (FWHM) measurements shown in Figures $5 \mathrm{~A}$ and $\mathrm{C}$.

A)

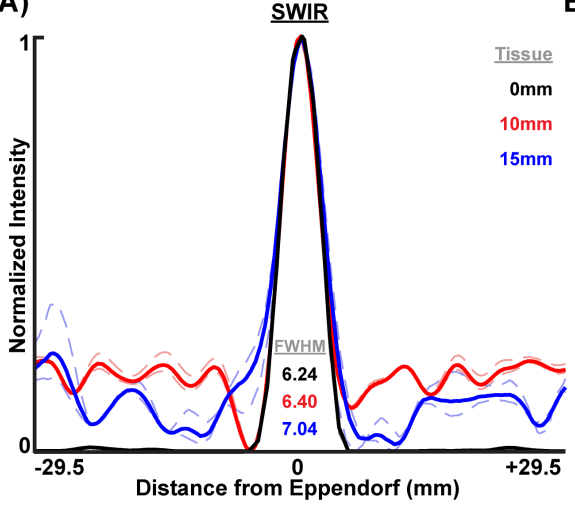

B)

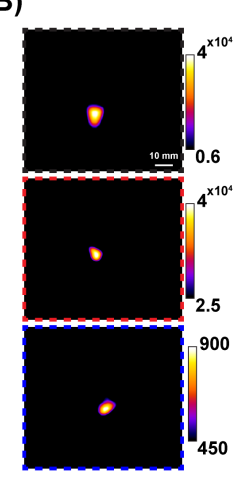

C)

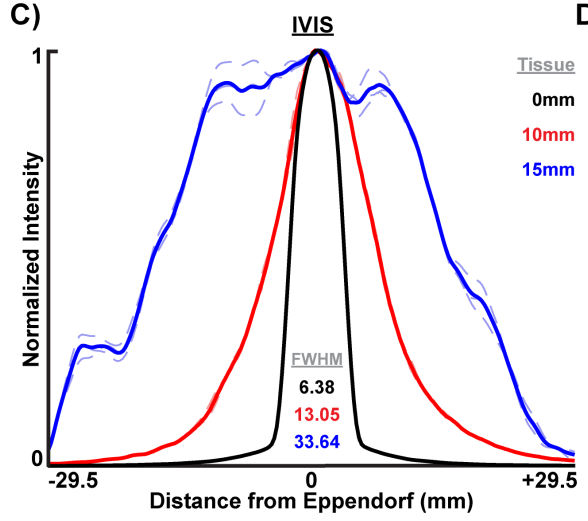

D)

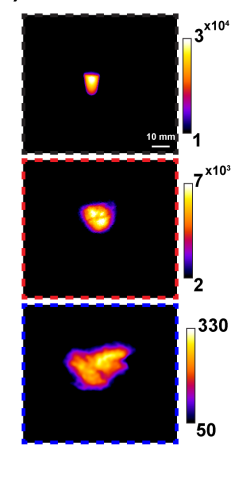

Figure 5. SWIR CLI enables reduced scattering in tissue over VIS CLI A) Normalized SWIR CLI intensity profiles of an eppendorf containing $1.5 \mathrm{mCi}$ of ${ }^{90} \mathrm{Y}$ suspended in $200 \mu \mathrm{l}$ of saline solution with increasing depths of scattering tissue (chicken breast at depths of 0, 10 and $15 \mathrm{~mm}$ ) with respective full width half maximums (FWHM) of 6.24, $6.40 \& 7.04 \mathrm{~mm}$. B) Representative SWIR CL images of the eppendorf through increasing scattering tissue depths. C) Normalized VIS CLI intensity profiles of VIS CLI line profiles (IVIS) from the phantom setup used in A). FWHMs increase with scattering tissue depth at 0, 10 and $15 \mathrm{~mm}$ with respective FWHMs of 6.38, 13.05 and $33.64 \mathrm{~mm}$. D) Representative VIS CLI images of the source as seen through scattering tissue 
depths. In all cases three seperate line measurements are made from the images at each depth (dotted lines) with the mean shown by the solid line.

\subsection{SWIR CLI radioisotope sensitivity limits in vitro}

As previously mentioned, the majority of SWIR sensors are insensitive to wavelengths of light below $920 \mathrm{~nm}$. However, by thinning the InP cap, the low cutoff can be improved to $\sim 400 \mathrm{~nm}$ with the sensor then capable of detecting VIS and SWIR wavelengths. Whilst the camera has a largely increased range, it is still very insensitive in comparison to e.g. EMCCDs. It should be noted that the VIS sensitivity of a second sensor (Zephir 1.7v, Photon Etc., Canada) used in this study has significantly reduced quantum efficiency in the VIS range (e.g. $\sim 25 \%$ at $600 \mathrm{~nm})$. The sensor was employed to compare the spatial localization of VIS-SWIR $(400-1700 \mathrm{~nm})$, NIR-SWIR $(650$ $1700 \mathrm{~nm}$ ) and SWIR CLI (900 - $1700 \mathrm{~nm}$ ) of radioisotopes via appropriate long pass optical filters. Silica nanoparticles (SiNPs) have been shown to readily bind radioisotopes with a high affinity and are suitable for localized injection in vivo preventing unwanted distribution of the radioisotope.[40] In Figure 6, ${ }^{68} \mathrm{Ga}$ conjugated SiNPs suspended in $30 \mu \mathrm{l}$ of saline opposite a nonradiolabeled SiNP control were imaged at VIS-SWIR, NIR-SWIR, and again at SWIR following numerous half-lives. As can be seen in Figure 6 A) - C), the spatial location of the detected CL signals through the defined spectrums are solely localized to the eppendorf containing ${ }^{68} \mathrm{Ga}+$ SiNPs. The overall activity along with the activity per $\mu$ lare shown. In Figure $6 \mathrm{D}$ ) the sensitivitiy of SWIR CLI is shown with a detection limit of $7 \mu \mathrm{Ci}(0.23 \mu \mathrm{Ci} / \mu \mathrm{l})$ for ${ }^{68} \mathrm{Ga}$ labelled SiNPs.
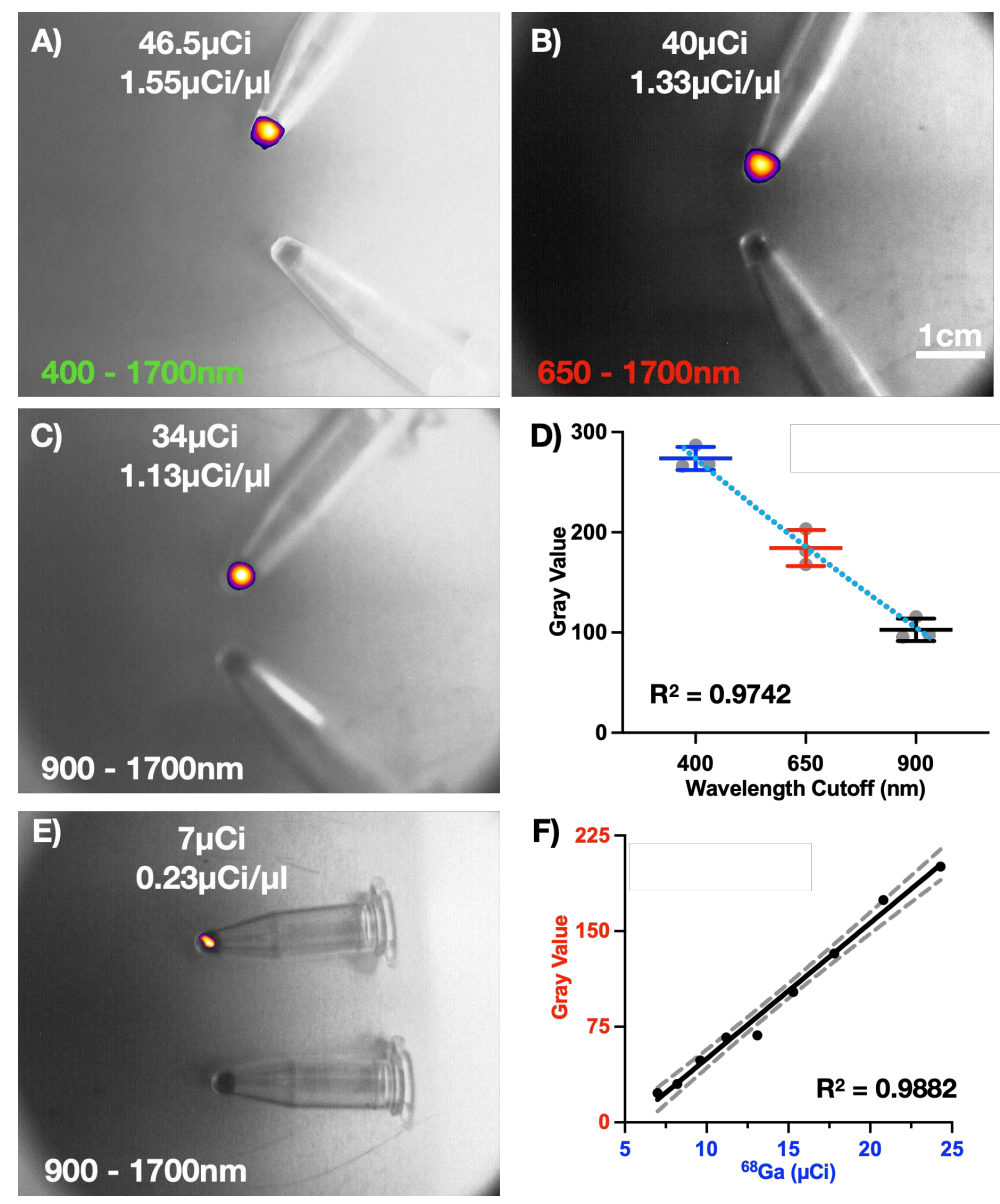
Figure 6. SWIR CLI radioisotope sensitivity limits for ${ }^{68} \mathrm{Ga}$ labeled SiNPs. A) VIS to SWIR $(400-1700 \mathrm{~nm})$ image of ${ }^{68} \mathrm{Ga}$ radiolabeled SiNPs (Top) and non-radiolabeled SiNPs (Bottom). B) NIR to SWIR (650 - $1700 \mathrm{~nm}$ ) image of the same phantom as in A). C) SWIR image of the same phantom as in A). D) Decay corrected emission profile quantification of the labeled ${ }^{68} \mathrm{Ga}$ SiNPs, $n=3$ technical replicates, median and standard deviation are shown. The dotted line represents a fitted one phase exponential decay with the goodness of fit shown $\left(R^{2}=0.9742\right)$. E) The SWIR CLI radioisotope sensitivity limit for ${ }^{68} \mathrm{Ga}$ labeled SiNPs post multiple half-lives. F) Tracking the decay for the SWIR CLI limit of detection (900nm LP Filter). The linear regression is shown with $95 \%$ confidence intervals with a goodness of fit $R^{2}=0.9882$, highlighting that at these low levels SWIR CLI performs linearly in relation to radioisotope levels.

\subsection{SWIR CLI radioisotope sensitivity limits ex vivo}

Having determined the in silico detection limits of SWIR CLI to be $7 \mu \mathrm{Ci}(0.23 \mu \mathrm{Ci} / \mu \mathrm{l})$ for ${ }^{68} \mathrm{Ga}$ for radiolabeled SiNPs, we then tested the detection limits in tissue. A nude mouse was euthanized via $\mathrm{CO}_{2}$ inhalation and injected with $30 \mu \mathrm{l}$ of ${ }^{68} \mathrm{Ga}$ labelled SiNPs into each respective paw with varying radioisotope activities. Confirmation of the SiNPs location can be seen in Figure $7 \mathrm{~A}$ acquired with the IVIS system (VIS CLI). The same mouse was then transferred to the same SWIR CLI system as used in Figure 6 and imaged post multiple half-lives to determine the post vivo sensitivity SWIR CLI sensitivity (900 nm LP filter). As can be seen in Figure 7B, ${ }^{68} \mathrm{Ga}$ labeled SiNPs were successfully detected down to $10.9 \mu \mathrm{Ci}$, far below activity levels administered clinically (typically $4000 \mu \mathrm{Ci}) \cdot[41]$
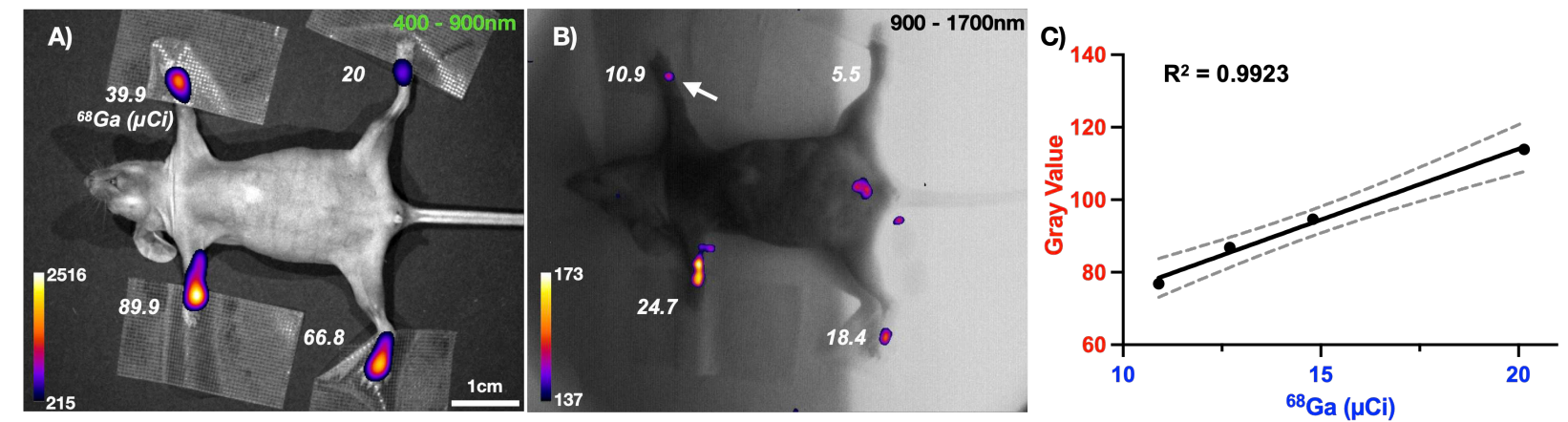

Figure 7. Ex vivo SWIR CLI radioisotope sensitivity limits A) VIS CLI (IVIS - 400 to $900 \mathrm{~nm}$ ) image of $30 \mu \mathrm{l}$ of ${ }^{68} \mathrm{Ga}$ labeled SiNPs injected subcutaneously into a dead mouse paw labeled with varying amounts of ${ }^{68} \mathrm{Ga} .{ }^{68} \mathrm{Ga}$ amounts are shown in white text in $\mu \mathrm{Ci}$. B) The ex vivo SWIR CLI (900- $1700 \mathrm{~nm}$ ) limit of detection for ${ }^{68} \mathrm{Ga}$ labeled SiNPs in the same mouse as in A) post multiple half-lives. As expected, the detection limit worsens in tissue compared to in silico imaging $\left(\sim 3 \mu \mathrm{Ci}\right.$ less sensitive). C) Linear regression analysis of the ex vivo SWIR CLI of the ${ }^{68} \mathrm{Ga}$ labeled SiNPs to the limit of detection $(10.9 \mu \mathrm{Ci}, \mathrm{B})$ paw labeled with the white arrow). SWIR CLI detection in tissue behaves linearly in relation to $\mu \mathrm{Ci}$ amounts $\left(R^{2}=0.9923\right)$.

\subsection{In vivo detection of radioisotope SWIR CLI}

Whilst ${ }^{68} \mathrm{Ga}$ produces bright $\mathrm{CL}$, the combination of it's short half-life (68 mins), the increased noise of current SWIR CLI system and increased y strikes rendered ${ }^{68} \mathrm{Ga}$ unsuitable for longer term experiments. To counteract the short comings of both ${ }^{68} \mathrm{Ga}$ and SWIR imaging we employed ${ }^{90} \mathrm{Y}$ (average $\beta$ energy of $0.94 \mathrm{MeV}, 64.1$ hour half-life) conjugated to SiNPs for in vivo SWIR CLI radioisotope detection.[40] Additionally, ${ }^{90} \mathrm{Y}$ has been shown to improve the SNR of CL detection 
as the produced $\beta^{-}$do not result in $511 \mathrm{keV}$ photon production as is found with $\beta^{+}$sources e.g. ${ }^{18} \mathrm{~F}$ or ${ }^{68} \mathrm{Ga}$.[42] The long exposure times for SWIR CLI enabled contamination of the SWIR CL image with a thermal signature, further complicating SWIR CLI in comparison to VIS CLI. Mice which had been injected with $\sim 200 \mu \mathrm{Ci}$ of ${ }^{90} \mathrm{Y}$ labeled SiNPs were imaged 3 hours post injection into a single footpad. ${ }^{90} \mathrm{Y}$ is administered clinically for radioembolization at activities ranging from 13,500 to $143,000 \mu \mathrm{Ci} .[38,39]$ As can be seen in Figure 8 and Suppelemental Figure 3 the SWIR CLI signal was readily detected over background thermal signatures present in vivo $(\mathrm{n}=4$ mice $)$. Radiolabeled SiNPs injected in the foot slowly migrate through the lymphatic system (on the order of $48 \mathrm{hrs}$ ) preventing $\mathrm{CL}$ contamination of the inherent thermal signature.[40] To highlight the SWIR CL signal, the respective thermal signature from each mouse was used to divide the image producing measurements in terms of signal to thermal background ratio (SBR). The performed image processing steps can be seen in Supplemental Figure 1. This processing method suppressed the inherent thermal signal in the control mouse, shown in Figure 8. As can be seen in Figure $8 \mathrm{~B}, \mathrm{SBRs}$ from injected mice ranged from 1.68 to 4.63 with a mean of 3.07 .

335

336

337

338

339

340

341
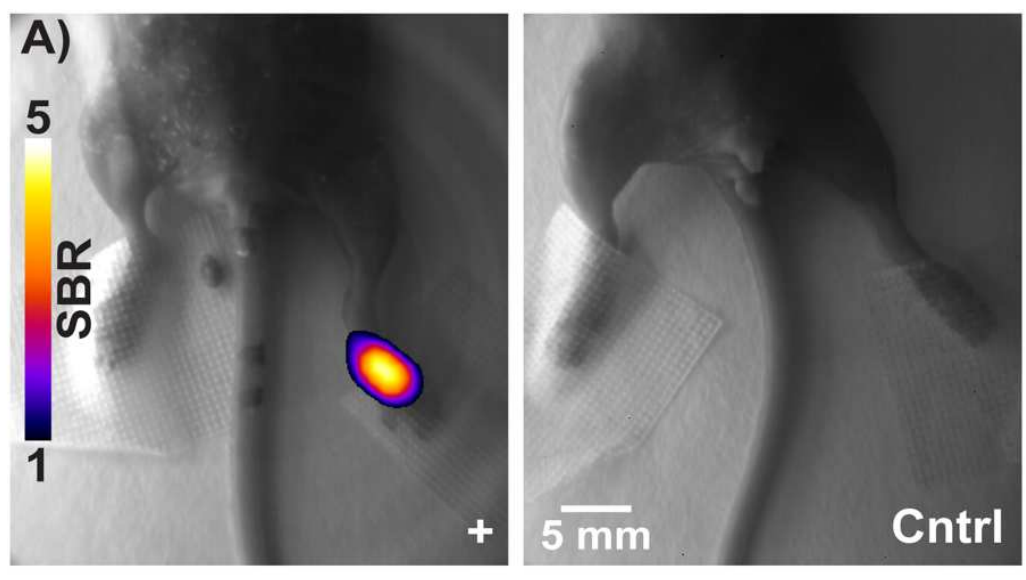

Figure 8. In vivo SWIR CLI detection of ${ }^{90} Y$ labeled SiNPs three hours post injection into the footpad A) Left, Representative images of a mouse injected (+) with ${ }^{90}$ Y labeled SiNPs ( 200 $\mu$ Ci). Right, image of a control mouse without any injection. C) Quantified SBR values of injected $(n=4)$ vs control mice $(n=1)$. All images are shown in respective signal to background ratios $(S B R)$.

\section{Discussion}

This work set out to prove that SWIR CLI could be detected from radioisotopes from commercially available SWIR technology. Previous work has shown SWIR CL produced by accelerated beams from LINAC sources, which have on average an order or more magnitude higher energy levels than $\beta$ particles from radioisotopes. $[23,26]$ The employed enclosure provided an imaging environment free of ambient room lights as is necessary with radioisotope VIS CLI.[43, 44] The TEC SWIR sensors used here, whilst state of the art, produce 2 to 3 orders of magnitude higher dark charge noise in comparison to e.g. TEC EMCCD counter parts. Despite this, SWIR CLI was detected from a variety of medical radioisotopes, see Figure 3A, and the detected SWIR radiance levels are in line with radioisotope production in VIS CLI.[34] We have also shown that in line with VIS CLI, SWIR CLI linearly correlated with both high and low radioisotope levels in multiple settings, see Figures 2, 6 and 7 . We have shown the radioisotope sensitivity limit of SWIR CLI to be as low as $7 \mu \mathrm{Ci}(0.23 \mu \mathrm{Ci} / \mu \mathrm{l})$ in vitro and as low as $10.9 \mu \mathrm{Ci}$ in tissue with ${ }^{68} \mathrm{Ga}$. This is in stark contrast to VIS CLI performed with EMCCDs where ${ }^{68} \mathrm{Ga}$ detection has been reported as low as $0.00009 \mu \mathrm{Ci} / \mu \mathrm{l}$, over four orders of 
magnitude more sensitive than SWIR CLI.[45] As a result, the insensitivity of SWIR CLI limits applications in its current iteration. However, the significant advantage of reduced scattering in SWIR CLI as shown here in Figure 5 could significantly improve radioisotope CLI applications.[26] Based on these results the advantage of radioisotope SWIR CLI would enable the better resolution of deep seated tumor radioisotope uptake.[17, 46, 47]

The SWIR CLI setup used here was capable of detecting radioisotope emissions at temporal resolutions of up to $0.25 \mathrm{~s}$, verging on the potential for video rate imaging, as shown in Figure 4A. However, ${ }^{32} \mathrm{P}$ and the amount of radioisotope used in this case is only suitable for in silico experimentation and enabled this proof of principle investigation. Furthermore, ${ }^{32} \mathrm{P}$ undergoes pure $\beta^{-}$decay reducing the impact of $y$ strikes on the sensor and improving sensitivity.[42] The long half-life of ${ }^{32} \mathrm{P}$, further enabled quantification of the radioisotope SWIR CL spectrum, found to be in line with the SWIR CL spectrum produced by LINACs.[26] Due to the inherent noise in SWIR systems, detection of CL could not be reliably determined from noise above $1400 \mathrm{~nm}$. A more sensitive system with reduced noise, similar to that of EMCCD cameras should be able to detect these longer wavelengths whilst simultaneously detecting lower radioactivity levels. However, the advantage of wavelengths above $1400 \mathrm{~nm}$ is unclear for SWIR CLI due to the increased absorption of water in this region.[17, 46]

Having performed SWIR CLI from a range of radioisotopes, determined the sensitivity limits and shown the advantage of radioisotope SWIR CLI over VIS CLI our focus then turned to the preclinical potential of SWIR CLI. The success of preclinical VIS CLI has been widely established due to its relative ease and the wide availability of highly sensitive (single photon) cameras.[4851] Initial experiments focused on ex vivo SWIR CLI to detect ${ }^{18} \mathrm{~F}$-FDG locally injected to a tumor on a xengofrafted mouse (see Supplemental Figure 2). However, the weak CL production of ${ }^{18} \mathrm{~F}$ (22 times dimmer than ${ }^{68} \mathrm{Ga}$ ) required the intratumoral administering of mCi's of radioisotope and lengthy acquisition times on the order of an hour for accurate signal detection.[45] ${ }^{90} \mathrm{Y}$ is a brighter $\mathrm{CL}$ source than ${ }^{68} \mathrm{Ga}$ with a longer half-life $(64.2 \mathrm{hrs}$ versus $1.13 \mathrm{hrs})$ and overcomes the limitations of ${ }^{68} \mathrm{Ga}$ and ${ }^{18} \mathrm{~F}$ for this investigation.[2, 34] ${ }^{90} \mathrm{Y}$ labeled SiNPs were synthesized as previously described and injected into the foot of live mice to enable in vivo SWIR CLI detection.[40] Four mice were imaged 3 hours post injection with $\sim 200 \mu \mathrm{Ci}$ of ${ }^{90} \mathrm{Y}$-SiNPs, respectively along with a non-injected control mouse. In this case, an imaging time of 15 mins provided a reliable signal that could be detected over the endogenous thermal signature, see Figure 8. This is the first case of radioisotope SWIR CLI in vivo detection and proves the potential for in vivo radioisotope SWIR CLI.

Throughout the course of these experiments the main limiting factor has been the intinsic noise within the SWIR setup, an obstacle not only for SWIR CLI.[52] The orders of magnitude noise increase compared to EMCCD detectors and reduced light output at these longer wavelengths requires longer exposure times (tens of minutes versus seconds to minutes). This long exposure time is a significant disadvantage for SWIR CLI and counteracts one of the benefits of CLI i.e. short acquisition times. The SWIR sensors used here are not capable of performing on chip binning and are limited to post processing binning, additionally reducing the sensitivity and acquisition speed when compared to EMCCDs. Further hampering signal detection is the limited commercial availability of efficient light collection (fast) SWIR lenses. In this case an f/1.4 was achieved whilst numerous VIS CLI spectrum lenses collect more than double the light with an f/0.95.[53-55] To counteract this we utilized lenses with a short focal length (8 or $16 \mathrm{~mm}$ ) to decrease the distance to the source, increasing light collection. However, imaging with such short focal lengths comes at the cost of reducing the effective depth of field, especially in comparison to the current preclinical gold standard IVIS imaging. Future iterations of SWIR CLI systems should aim to tackle these two main current limitations with a faster lens and dark noise reduced 
camera sensor. Such a lens and sensor will be highly custom devices and are outside the scope of this proof of principle work.

Considering that human eyes respond to light from $\sim 400$ to $\sim 700 \mathrm{~nm}$ it would be feasible to change the lights used in CLI locations from bulbs which produce SWIR light to non-SWIR emitting LEDs. The use of such room lighting in combination with SWIR CLI could enable radioisotope CLI to be carried out in a well lit room and without the need for a dark enclosure, as has been achieved for LINAC CLI.[28] This may have impact in preclinical small animal imaging where CLI is commonly used as a cheap alternative to PET for the tracking of novel radiotracers and treatments.[49, 56] However, significant improvements are required in SWIR optics and technology before this can be realized.

\section{Methods \& Materials}

\subsection{Radioisotope SWIR Setup}

Detection of SWIR CLI was carried out using thermoelectronically cooled (TEC) InGaAs focal plane arrays (NIRVana 640 TE, Princeton Teledyne, NJ, USA or ZephIR 1.7x, Photon etc Inc., Montreal, Canada) mounted on a custom enclosure as outlined in Figure 1. The custom built aluminium enclosure has an adjustable stage and lockable door. SWIR CL collection was achieved via a SWIR lens (SWIR-16, Navitar, NY, USA or 8mm FL SWIR lens, stock \#83-815, Edmund Optics, NJ, USA) mounted on the camera. In all cases the lens aperture was set to an $f / 1.4$ to allow maximum collection of light. Data presented in Figures 2, 3 and 4 were acquired without the addition of optical filters, the NIRVana 640 TE focal plane array used in these acquisitions has not had InP cap layer thinning performed, preventing it detecting photons below $920 \mathrm{~nm}$.[57] Data in all other figures was acquired with the addition of either an 650nm or $900 \mathrm{~nm}$ O.D. 4.0 long pass filter (Edmund Optics \#84-759 \& \#84-764, NJ, USA) mounted to the front of the lens. Acquisition settings along with recording of data was controlled via each cameras respective acquisition software. Due to the high noise levels associated with InGaAs sensors in comparison to more developed technology such as EMCCDs, acquisitions were limited to an accumulation of 90 frames of 10 s each $(900 \mathrm{~s} / 15$ mins total recording time). This ensured that Cerenkov light could be detected over the fundamental noise in the system. Dark noise measurements were recorded for each sensor and subtracted from the SWIR CL signal. White light (WL) images were taken with the enclosure door open and room lights on.

\subsection{Determining SWIR CLI Sensitivity}

Radioisotopes were located within either plastic Eppendorf's or glass scintillation vials and imaged with the lids off in the enclosure, whilst being held in a suitable lead pig to limit exposure. White light images were acquired with the door open and room lights on (fluorescent tube bulbs with SWIR spectral emission (Pentron 3000K, Osram Sylvania, MA, USA)).[58] Corresponding SWIR CLI was performed after closing the door and adjusting the exposure time to $10 \mathrm{~s}$. Black posterboard (TB5, Thorlabs, NJ, USA) was placed over the source to prevent propagation of light whilst allowing $\mathrm{Y}$ particles to pass freely.

\subsection{Determining the radioisotope SWIR CLI temporal resolution and emission spectrum}

${ }^{32} \mathrm{P}$ ( $\beta$ energy average $0.695 \mathrm{MeV}, \mathrm{t}_{1 / 2} 14.3$ days) was used to determine the temporal resolution limits of the setup along with the SWIR CL emission spectrum. The long half-life of the ${ }^{32} \mathrm{P}$ provided a constant photon flux. Approximately $10 \mathrm{mCi}$ of ${ }^{32} \mathrm{P}$ (NEX060005MC, Perkin Elmer, MA, USA) in $1 \mathrm{ml}$ of water was imaged in the setup as previously outlined. The limit of temporal 
acquisition was determined by acquiring 90 frames at a range of exposure times from 10 to $0.1 \mathrm{~s}$. The limit of detection was defined as a level in which the source could not be visually determined from the inherent noise in the system. The SWIR CL spectrum was determined using appropriate long pass (LP) filters separated by $100 \mathrm{~nm}$ ranging from 1000 to $1500 \mathrm{~nm}$ (FELH1000:100:1500, Thorlabs, NJ, USA) mounted on the front of the lens (SM1L03 and SM1A57, Thorlabs, NJ, USA). Acquisitions were performed as outlined previously.

\subsection{Image processing and statistical analysis}

Image processing was carried out with the open source software Fiji (ImageJ 2.0, [59]). Processing steps are outlined in Suppelementary Figure 1. Briefly, darknoise levels acquired with the same settings and conditions were subtracted from the data. Binning (8x8) was applied to improve sensitivity along with median filtering was applied to remove y strikes. Artifacts were further removed via FFT transformations and ImageJ rolling ball background subtraction.[60] Finally, the image was resized for overlay with the white light image. Reported values were collected using ROI measurements in Fiji. Statistical analysis and graphing were carried out in GraphPad Prism9 (GraphPad Software LLC, CA, USA). Information on statistical analyses is given for each figure along with technical and biological replicate numbers. SWIR radiance of respective isotopes was calculated by recording gray values and correcting for isotope concentration $(\mu \mathrm{Ci} / \mu \mathrm{l})$ and FOV $\left(\mathrm{cm}^{2}\right)$. Full width half maximum measurements were carried out using custom code in MATLAB (2020b, Mathworks Inc., USA).

\subsection{Silica nanoparticle radiolabeling and injection}

Silica nanoparticles were labeled with either ${ }^{68} \mathrm{Ga}$ or ${ }^{90} \mathrm{Y}$ as previously described.[40] Briefly, silica nanoparticles (SiNPs) were incubated with free isotope at a $\mathrm{pH}$ of 8.8 for 60 mins, on a thermomixer at $70^{\circ} \mathrm{C}$ and $500 \mathrm{rpm}$. After completion of the labeling protocol, radiolabeled SiNPs were resuspended in $30 \mu \mathrm{l}$ of saline solution for injection into the footpad.

\subsection{Preclinical SWIR CLI}

All handling and mouse experiments were carried out in accordance with Institutional Animal Care and Use Committee (IACUC) guidelines at MSKCC and the NIH Guide for the Care and Use of Laboratory Animals. In vivo animal procedures were performed under anesthesia by inhalation of $3 \%$ isoflurane in $100 \% \mathrm{O}_{2} \mathrm{v} / \mathrm{v}$ followed by $1-2 \%$ isoflurane in $100 \% \mathrm{O}_{2} \mathrm{v} / \mathrm{v}$ for maintenance. All mice $\left(n=13\right.$ in total, FoxN1 ${ }^{\mathrm{NU}}$, Stock \#069, Envigo, USA) were housed under appropriate conditions with food and water ad libitum. Anesthetized mice $(n=4)$ were xenografted via injection with $1 \times 10^{6} 4 \mathrm{~T} 1$ cells (ATCC, CRL-2539) suspended in $30 \mu \mathrm{l}$ of Matrigel (Corning, \#354234) into the fourth mammary pad. Tumors were allowed to proliferate for 2 weeks before being imaged. In all cases mouse euthanasia was performed using $\mathrm{CO}_{2}$ in accordance with approved protocols. For Supplementary Figure 2, following euthanasia, $\mathrm{n}=3$ xenografted mice were injected (blinded) with up to $4.5 \mathrm{mCi}$ of ${ }^{18} \mathrm{~F}$-FDG with one mouse not receiving any injection (negative control). Additionally, $\mathrm{n}=1$ euthanized mouse was injected into the footpad with ${ }^{68} \mathrm{Ga}$ labeled SiNPs. Finally, $n=4$ mice were injected with ${ }^{90} Y$ labeled SiNPs with an additional $n=1$ mouse not receiving any injection and providing a negative control for in vivo experiments.

\section{Acknowledgements}

We would like to thank Craig Wall, Manjul Shah, Sabbir Liakat and Princeton Teledyne for providing the NIRvana 640 TE camera and lens along with Photon Etc. for providing the Zephir $1.7 x$ camera. Sincere thanks go to Valerie Longo and Pat Zanzonico of the small animal imaging 
511

512

513

514

515

516

517

518

519

520

521

522

523

524

525

526

527

528

529

530

531

532

533

534

535

536

537

538

539

540

541

542

543

544

545

546

547

548

549

550

551

552

553

554

facility at MSKCC. This work was funded by the National Institutes of Health $\mathrm{NCl}$ grants R01CA183953 (to Jan Grimm) and P30 CA08748 (to Craig Thompson/MSKCC).

\section{Conflict of Interests}

The authors declare no conflicts of interest.

\section{Author Contributions}

BML setup the system, designed and carried out experiments, recorded images and carried out the data analysis. QZ performed the radiolabeling of SiNPs. MS, EI, $\mathrm{HH}$ \& $\mathrm{AO}$ aided in vivo experiments. BML, ECP \& JG designed the study. All authors aided with the experimental setup, experimental procedures, data interpretation, data presentation and writing of the manuscript.

\section{References}

1. Čerenkov, P. Visible light from pure liquids under the impact of $\gamma$-rays. in CR (Dokl.) Acad. Sci. URSS. 1934.

2. Ciarrocchi, E. and N. Belcari, Cerenkov luminescence imaging: physics principles and potential applications in biomedical sciences. EJNMMI physics, 2017. 4(1): p. 1-31.

3. Glaser, A.K., et al., Cherenkov radiation fluence estimates in tissue for molecular imaging and therapy applications. Physics in Medicine \& Biology, 2015. 60(17): p. 6701.

4. Ruggiero, A., et al., Cerenkov luminescence imaging of medical isotopes. Journal of Nuclear Medicine, 2010. 51(7): p. 1123-1130.

5. Contalbrigo, M., et al., The CLAS12 ring imaging Cherenkov detector. Nuclear Instruments and Methods in Physics Research Section A: Accelerators, Spectrometers, Detectors and Associated Equipment, 2020: p. 163791.

6. Mitchell, G.S., et al., In vivo Cerenkov luminescence imaging: a new tool for molecular imaging. Philosophical Transactions of the Royal Society A: Mathematical, Physical and Engineering Sciences, 2011. 369(1955): p. 4605-4619.

7. Tamura, R., E.C. Pratt, and J. Grimm. Innovations in nuclear imaging instrumentation: Cerenkov imaging. in Seminars in nuclear medicine. 2018. Elsevier.

8. Miao, T., et al., Cherenkov imaging for linac beam shape analysis as a remote electronic quality assessment verification tool. Medical physics, 2019. 46(2): p. 811-821.

9. D'Souza, J.W., et al., Cerenkov luminescence imaging as a modality to evaluate antibodybased PET radiotracers. Journal of Nuclear Medicine, 2017. 58(1): p. 175-180.

10. Olde Heuvel, J., et al., (68)Ga-PSMA Cerenkov luminescence imaging in primary prostate cancer: first-in-man series. European journal of nuclear medicine and molecular imaging, 2020. 47(11): p. 2624-2632.

11. Thorek, D.L., C.C. Riedl, and J. Grimm, Clinical Cerenkov luminescence imaging of 18F-FDG. Journal of Nuclear Medicine, 2014. 55(1): p. 95-98.

12. Grootendorst, M.R., et al., Intraoperative assessment of tumor resection margins in breast-conserving surgery using 18F-FDG Cerenkov luminescence imaging: a first-inhuman feasibility study. Journal of Nuclear Medicine, 2017. 58(6): p. 891-898. 
13. Jarvis, L.A., et al., Cherenkov Video Imaging Allows for the First Visualization of Radiation Therapy in Real Time. International Journal of Radiation Oncology*Biology*Physics, 2014. 89(3): p. 615-622.

14. Jacques, S.L., Optical properties of biological tissues: a review. Physics in Medicine \& Biology, 2013. 58(11): p. R37.

15. Yaroshevsky, A., et al., Transition from the ballistic to the diffusive regime in a turbid medium. Optics letters, 2011. 36(8): p. 1395-1397.

16. Ding, F., et al., Recent advances in near-infrared II fluorophores for multifunctional biomedical imaging. Chemical science, 2018. 9(19): p. 4370-4380.

17. Cao, Q., et al., Multispectral imaging in the extended near-infrared window based on endogenous chromophores. Journal of biomedical optics, 2013. 18(10): p. 101318.

18. Thorek, D.L., et al., Quantitative imaging of disease signatures through radioactive decay signal conversion. Nature medicine, 2013. 19(10): p. 1345.

19. Zhang, Q., et al., Ultrasmall Downconverting Nanoparticle for Enhanced Cerenkov Imaging. Nano Letters, 2021. 21(10): p. 4217-4224.

20. Pratt, E.C., et al., Nanoparticles as multimodal photon transducers of ionizing radiation. Nature nanotechnology, 2018. 13(5): p. 418-426.

21. Thimsen, E., B. Sadtler, and M.Y. Berezin, Shortwave-infrared (SWIR) emitters for biological imaging: a review of challenges and opportunities. Nanophotonics, 2017. 6(5): p. 1043-1054.

22. Carr, J.A., et al., Shortwave infrared fluorescence imaging with the clinically approved near-infrared dye indocyanine green. Proceedings of the National Academy of Sciences, 2018. 115(17): p. 4465-4470.

23. Naczynski, D.J., et al., X-ray-induced shortwave infrared biomedical imaging using rareearth nanoprobes. Nano letters, 2015. 15(1): p. 96-102.

24. Cao, X., et al., Cherenkov excited short-wavelength infrared fluorescence imaging in vivo with external beam radiation. Journal of biomedical optics, 2018. 24(5): p. 051405.

25. Dothager, R.S., et al., Cerenkov radiation energy transfer (CRET) imaging: a novel method for optical imaging of PET isotopes in biological systems. PloS one, 2010. 5(10): p. e13300.

26. Cao, X., et al., Observation of short wavelength infrared (SWIR) Cherenkov emission. Optics letters, 2018. 43(16): p. 3854-3857.

27. Glaser, A.K., et al., Optical dosimetry of radiotherapy beams using Cherenkov radiation: the relationship between light emission and dose. Physics in Medicine \& Biology, 2014. 59(14): p. 3789.

28. Glaser, A.K., et al., Time-gated Cherenkov emission spectroscopy from linear accelerator irradiation of tissue phantoms. Optics Letters, 2012. 37(7): p. 1193-1195.

29. Browne, E., Commonly used radioactive sources. The European Physical Journal. C, Particles and Fields., 2000. 15(1-4): p. 190-190.

30. Pritychenko, B., et al., The nuclear science references (NSR) database and web retrieval system. Nuclear Instruments and Methods in Physics Research Section A: Accelerators, Spectrometers, Detectors and Associated Equipment, 2011. 640(1): p. 213-218.

31. Tendler, I.I., et al., Experimentally observed cherenkov light generation in the eye during radiation therapy. International Journal of Radiation Oncology* Biology* Physics, 2020. 106(2): p. 422-429. 
32. Darr, C., et al., Intraoperative 68Ga-PSMA Cerenkov luminescence imaging for surgical margins in radical prostatectomy: a feasibility study. Journal of Nuclear Medicine, 2020. 61(10): p. 1500-1506.

33. Banerjee, S.R. and M.G. Pomper, Clinical applications of Gallium-68. Applied Radiation and Isotopes, 2013. 76: p. 2-13.

34. Beattie, B.J., et al., Quantitative modeling of Cerenkov light production efficiency from medical radionuclides. PloS one, 2012. 7(2): p. e31402.

35. Martin, T. and P. Dixon, InGaAs sees infrared and visible light. Laser focus world, 2004. 40(11): p. 109-111.

36. Williamson, J.B., et al., High-density, planar Zn-diffused InGaAs/InP photodetector arrays with extended short-wavelength response. IEEE Transactions on Electron Devices, 1991. 38(12): p. 2707.

37. Hoelter, T.R. and J.B. Barton. Extended short-wavelength spectral response from InGaAs focal plane arrays. in Infrared Technology and Applications XXIX. 2003. International Society for Optics and Photonics.

38. Kim, S.P., et al., A guide to 90Y radioembolization and its dosimetry. Physica Medica, 2019. 68: p. 132-145.

39. Fidelman, N., et al., Radioembolization with 90Y glass microspheres for the treatment of unresectable metastatic liver disease from chemotherapy-refractory gastrointestinal cancers: final report of a prospective pilot study. Journal of gastrointestinal oncology, 2016. 7(6): p. 860.

40. Shaffer, T.M., et al., Silica nanoparticles as substrates for chelator-free labeling of oxophilic radioisotopes. Nano letters, 2015. 15(2): p. 864-868.

41. Demirci, E., et al., Estimation of the organ absorbed doses and effective dose from 68GaPSMA-11 PET scan. Radiation protection dosimetry, 2018. 182(4): p. 518-524.

42. Carpenter, C.M., et al., Cerenkov luminescence endoscopy: improved molecular sensitivity with B--emitting radiotracers. Journal of Nuclear Medicine, 2014. 55(11): p. 1905-1909.

43. Alsheikh, H., [P091] Investigation of cherenkov imaging using IVIS bioluminescence scanner. Physica Medica: European Journal of Medical Physics, 2018. 52: p. 127.

44. Spinelli, A.E., et al., Multispectral Cerenkov luminescence tomography for small animal optical imaging. Optics Express, 2011. 19(13): p. 12605-12618.

45. de Wit-van der Veen, B., et al., Performance evaluation of Cerenkov luminescence imaging: a comparison of 68Ga with 18F. EJNMMI physics, 2019. 6(1): p. 1-13.

46. Carr, J.A., et al., Absorption by water increases fluorescence image contrast of biological tissue in the shortwave infrared. Proceedings of the National Academy of Sciences, 2018. 115(37): p. 9080-9085.

47. Zhang, H., et al., Penetration depth of photons in biological tissues from hyperspectral imaging in shortwave infrared in transmission and reflection geometries. Journal of biomedical optics, 2016. 21(12): p. 126006.

48. Balkin, E.R., et al., In vivo localization of $90 \mathrm{Y}$ and $177 \mathrm{Lu}$ radioimmunoconjugates using Cerenkov luminescence imaging in a disseminated murine leukemia model. Cancer research, 2014. 74(20): p. 5846-5854. 
49. Liu, M., et al., Cerenkov luminescence imaging on evaluation of early response to chemotherapy of drug-resistant gastric cancer. Nanomedicine: Nanotechnology, Biology and Medicine, 2018. 14(1): p. 205-213.

50. Zhang, Z., et al., Endoscopic Cerenkov luminescence imaging and image-guided tumor resection on hepatocellular carcinoma-bearing mouse models. Nanomedicine: Nanotechnology, Biology and Medicine, 2019. 17: p. 62-70.

51. Zhao, S., et al., Cerenkov luminescence imaging is an effective preclinical tool for assessing colorectal cancer PD-L1 levels in vivo. 2020.

52. Fraenkel, R., et al. Development of low-SWaP and low-noise InGaAs detectors. in Infrared Technology and Applications XLIII. 2017. International Society for Optics and Photonics.

53. Hu, Z., et al., Construction of a Novel Intraoperative Cerenkov luminescence Imaging System for Image-Guided Resection of Colorectal Cancer on Patients. Journal of Nuclear Medicine, 2017. 58(supplement 1): p. 527-527.

54. Grootendorst, M. and A. Purushotham, Clinical feasibility of intraoperative 18F-FDG Cerenkov Luminescence Imaging in breast cancer surgery. Journal of Nuclear Medicine, 2015. 56(supplement 3): p. 13-13.

55. Zhang, Z., et al., A novel in vivo Cerenkov luminescence image-guided surgery on primary and metastatic colorectal cancer. Journal of biophotonics, 2020. 13(3): p. e201960152.

56. $\mathrm{Xu}, \mathrm{Y}$., et al., Proof-of-concept study of monitoring cancer drug therapy with Cerenkov luminescence imaging. Journal of Nuclear Medicine, 2012. 53(2): p. 312-317.

57. Huang, Y.-H., et al., 10-Gb/s InGaAs pin photodiodes with wide spectral range and enhanced visible spectral response. IEEE Photonics Technology Letters, 2007. 19(5): p. 339-341.

58. Elvidge, C.D., et al., Spectral identification of lighting type and character. Sensors, 2010. 10(4): p. 3961-3988.

59. Schindelin, J., et al., Fiji: an open-source platform for biological-image analysis. Nature methods, 2012. 9(7): p. 676-682.

60. Walter, J., FFT-filter. Available at: http://rsb.info.nih.gov/ii/plugins/fft-filter.html., 2001. 


\section{Supplementary Files}

This is a list of supplementary files associated with this preprint. Click to download.

- SWIRCLISUPP21.pdf 